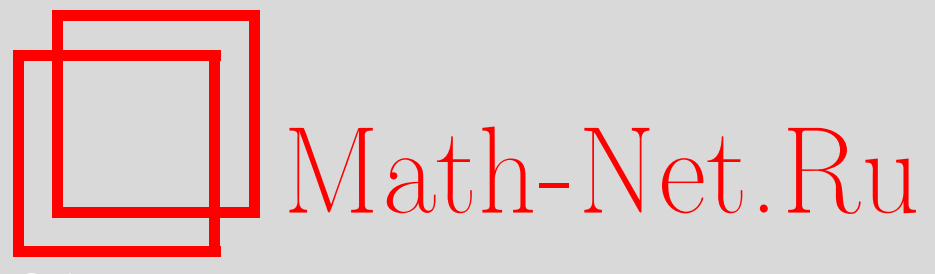

А. П. Котенко, М. Б. Букаренко, Система массового обслуживания с различимыми каналами как конечный автомат, Вестн. Сам. гос. техн. ун-та. Сер. Физ.мат. науки, 2012, выпуск 3(), 114-124

DOI: https://doi.org/10.14498/vsgtu1105

Использование Общероссийского математического портала Math-Net.Ru подразумевает, что вы прочитали и согласны с пользовательским соглашением

http://www . mathnet.ru/rus/agreement

Параметры загрузки:

IP : 54.81 .137 .203

26 апреля 2023 г., 11:34:35

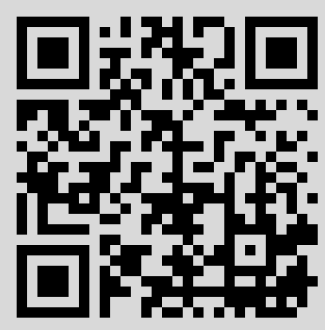


Вестн. Сам. гос. техн. ун-та. Сер. Физ.-мат. науки. 2012. № 3 (28). С. 114-124

УДК 519.248, 519.713.2

\title{
СИСТЕМА МАССОВОГО ОБСЛУЖИВАНИЯ С РАЗЛИЧИМЫМИ КАНАЛАМИ КАК КОНЕЧНЫЙ АВТОМАТ
}

\author{
А. П. Котенко, М. Б. Букаренко \\ Самарский государственный технический университет, \\ 443100, Россия, Самара, ул. Молодогвардейская, 244. \\ E-mails: ako1959@mail.ru, maxim.bukarenko@gmail.com
}

\begin{abstract}
Рассматриваются системы массового обслуживания с различимыми каналами, имеющими разную пропускную способность или раздельные очереди. Введён протокол диспетчеризачии входных заявок для минимизации среднего времени обслуживания заявок и вероятности отказа. Такие системы рассматриваются как детерминированные или недетерминированные конечные автоматы с уравнениями состояния в виде полиномов Жегалкина.
\end{abstract}

Ключевые слова: система массового обслуживания, конечный автомат, различимье каналь, протокол диспетчеризации.

Актуальность и постановка задачи исследования. Целью настоящей работы является разработка описания и имитационного моделирования систем массового обслуживания (CMO) с неоднородными приборами с раздельными накопителями с запретом передачи заявок из одного накопителя в другой. В то время как большинство СМО, встречающихся на практике, можно отнести к системам с раздельными очередями, их моделированию посвящено лишь несколько работ.

Для систем с многосекционной памятью в [1] моделируется 4-канальная $\mathrm{CMO} \mathrm{с} \mathrm{двумя} \mathrm{типами} \mathrm{приборов} \mathrm{(по} \mathrm{две} \mathrm{пары} \mathrm{однородных} \mathrm{приборов).} \mathrm{Каждая}$ заявка обслуживается обоими типами приборов; выбор прибора из пары однородных случаен. При этом отсутствует постановка задачи в формулировке как теории массового обслуживания (ТМО), так и математической статистики, например, отсутствуют данные о законе распределения.

В работе [2] отмечена актуальность исследования СМО с раздельными очередями к приборам при различных дисциплинах обслуживания, а также тот факт, что аналитические методы ТМО плохо распространяются на системы данного типа, особенно в случае большого числа приборов $N$, когда использование численных методов затруднительно. Авторы рассматривают систему с $N$ приборами и диспетчеризацией, которая предполагает, что приборы имеют одинаковую производительность, т.е. однородны.

В работе [3] рассматривается подобная система с $N$ приборами с раздельными очередями, однако заявки представляются в форме сообщения из $n$ пакетов, каждый из которых посылается на случайно выбранный прибор, что также не соответствует общему случаю, рассматриваемому в настоящей статье. В более общем случае $[4,5]$ система с раздельными очередями рассматривалась в контексте исследования приоритетного обслуживания.

Наиболее полное развитие аналитические модели СМО с неоднородными

Андрей Петрович Котенко (к.ф.-м.н., доц.), доцент, каф. прикладной математики и информатики. Максим Борисович Букаренко, аспирант, каф. прикладной математики и информатики. 
приборами и общей очередью получили в работах В. В. Рыкова и Д. В. Ефросинина в рамках общей теории управляемых систем обслуживания. В работе [6] ставится задача существования оптимальной политики с точки зрения динамического программирования, однако в [7] показана неполнота этого доказательства.

Системы массового обслуживания с раздельными очередями и неоднородными приборами удалось аналитически смоделировать только в случае либо детерминированного входящего потока, либо «упорядоченного входа», когда заявка встаёт в очередь на обслуживание к первому прибору со свободным местом в очереди [8,9]. В таких системах диспетчеризация не зависит от состояния системы в момент поступления заявки. Таким образом, очевидна необходимость исследования СМО с различимыми каналами, наиболее точно отражающими реальные процессы, которые не всегда подчиняются аксиоматике процессов гибели и размножения.

В нотификации $[10,11]$ рассмотрим систему массового обслуживания с различимыми каналами (имеющими, к примеру, разную пропускную способность или раздельные очереди) сигнатуры $T=T\left(\mu_{1}, \mu_{2}, \ldots, \mu_{k} ; m_{1}, m_{2}, \ldots\right.$, $\left.m_{k}\right)$, где $\mu_{i}$ - пропускная способность, $m_{i}$ - число мест в очереди $i$-того канала, $i \in \overline{1, k} ; k>0$. Оптимизация работы системы по среднему времени обслуживания заявок и минимизации вероятности отказа достигается с помощью следующего протокола диспетчеризации входных заявок.

Пусть очередная входная заявка обнаруживает систему в состоянии $\left(x_{1}, x_{2}, \ldots, x_{k} ; y_{1}, y_{2}, \ldots, y_{k}\right)$, не являющемся состоянием отказа $(1,1, \ldots, 1$; $\left.m_{1}, m_{2}, \ldots, m_{k}\right)$, где $x_{i}=1$, если $i$-тый канал занят, $x_{i}=0$, если свободен, $y_{i}$ соответствует наполненности очереди этого канала. В первом случае, если существует один и только один канал (с номером $i$ ), способный принять заявку $\left(0 \leqslant y_{i} \leqslant\left|x_{i} m_{i}-1\right|\right)$, то заявка направляется к нему. В противном случае оптимальным считаем выбор $i$-того канала обслуживания, способного обработать заявку с минимальным средним суммарным временем $T$ обслуживания попавших в него заявок:

$$
T=\min _{i: 0 \leqslant y_{i} \leqslant\left|x_{i} m_{i}-1\right|} \mu_{i}^{-1}\left(y_{i}+1+\chi_{i}\right),
$$

где $\chi_{i}$ - случайная величина, характеризующая незавершённость обработки заявки, находящейся в $i$-том канале в момент поступления новой заявки, $0 \leqslant$ $\chi_{i} \leqslant 1$. Для простоты примем $\chi_{i}=1$.

СMO с детерминированной диспетчеризацией и недетерминированной выработкой сигналов на освобождение приборов. Далее рассмотрим двухканальную СМО с различимыми каналами пропускной способности $\mu_{1}>\mu_{2}$ без очереди (сигнатура $T=T\left(\mu_{1}, \mu_{2} ; 0,0\right)$ в нотификации $\left.[1,2]\right)$. Представим её поведение в дискретном времени $n \in \overline{0,+\infty}$ через недетерминированный конечный автомат (HKA) $K(S, A)$ с алфавитом внутренних состояний $S=$ $=\{(00),(01),(10),(11)\}$ без выделенных начального и конечного состояний, входным алфавитом $A=\{0,1\}$ и пустым выходным алфавитом. Буква 1 входного алфавита $A$ соответствует приходу заявки в систему, а 0 - выработке сигнала освобождения заявки одним из каналов. Матрица переходов автомата $K(S, A)$ с учётом возможной диспетчеризации (1) и недетерминированного выбора переходов $(11) \rightarrow(10)$ и $(11) \rightarrow(01)$ приведена в табл. 1. 
Таблица 1

Матрица переходов НKА $\boldsymbol{K}(\boldsymbol{S}, \boldsymbol{A})$ с четырьмя стохастическими дугами

\begin{tabular}{|c|c|c|c|}
\hline$S \backslash A$ & \multicolumn{2}{|c|}{0} & \multicolumn{2}{|c|}{1} \\
\hline \multirow{2}{*}{$(00)$} & \multirow{2}{*}{$(00)$} & $(10)$ & $(01)$ \\
\cline { 3 - 4 } & \multicolumn{2}{|c|}{$p_{1}$} & $p_{2}$ \\
\hline$(01)$ & \multicolumn{2}{|c|}{$(00)$} & $(11)$ \\
\hline$(10)$ & \multicolumn{2}{|c|}{$(00)$} & \multicolumn{2}{|c|}{$(11)$} \\
\hline \multirow{2}{*}{$(11)$} & $(10)$ & $(01)$ & \multicolumn{2}{|c}{$(11)$} \\
\cline { 2 - 3 } & $q_{1}$ & $q_{2}$ & \multicolumn{2}{|c}{} \\
\hline
\end{tabular}

Здесь $p_{1}$ - вероятность перехода по стохастической дуге $(00) \rightarrow(10)$ в соответствии с оптимальной диспетчеризацией (1), $p_{2}=1-p_{1}$ - дополнительная вероятность перехода по стохастической дуге $(00) \rightarrow(01)$. При этом входная заявка, заставшая систему в состоянии простоя обоих каналов (00), выбирает каналы 1 или 2 с частотами $p_{1}>0$ и $p_{2}=1-p_{1}>0$ соответственно. Аналогично, $q_{1}$ - вероятность перехода по стохастической дуге $(11) \rightarrow(10)$ при освобождении заявки каналом $1, q_{2}=1-q_{1}$ - дополнительная вероятность перехода по стохастической дуге $(11) \rightarrow(01)$ при освобождении заявки каналом 2. При этом уход обработанной (выходной) заявки из системы, находившейся в состоянии отказа (10), переводит систему в состояния (10) или (01) с частотами $q_{1}>0$ и $q_{2}=1-q_{1}>0$ соответственно. Остальные дуги орграфа нестохастические; переход по ним осуществляется при поступлении соответствующего входного сигнала.

На рис. 1 представлен граф переходов $\operatorname{HKA} K(S, A)$ при детерминированной оптимальной диспетчеризации (1). Здесь и на других рисунках разметкой $\alpha: \beta$ помечены стохастические дуги, переход по которым происходит с вероятностью $\beta$ при получении входного сигнала $\alpha$. Детерминированные петли описывают следующие переходы:

$(11) \rightarrow(11)$ - приход входной заявки (входного сигнала 1) в систему, находящуюся в состоянии отказа (11);

$(00) \rightarrow(00)-$ приход входного сигнала 0 в систему, находящуюся в состоянии простоя (00).

Второй вариант можно считать «холостым ходом» в работе СМО, которая была способна совершить обработку выходной заявки, но таковой не оказалось на месте в данный момент времени.

В случае недетерминированной оптимизации (1), допускающей переход части входных заявок по неоптимальной дуге $(00) \rightarrow(01)$, уравнения состояний $S(n+1)=\left(s_{1}(n+1), s_{2}(n+1)\right)$ автомата $K(S, A)$ получим из таблицы истинности булевых функций $s_{1}(n+1)$ и $s_{2}(n+1)$ (табл. 2$)$.

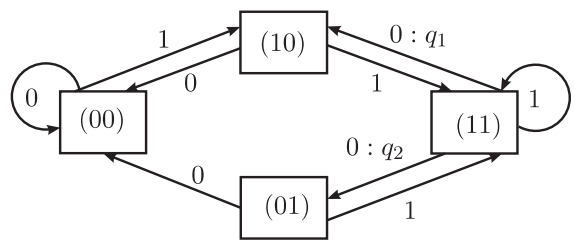

Рис. 1. Граф переходов НКА $K(S, A)$ с двумя стохастическими дугами $(11) \rightarrow(10)$ и $(11) \rightarrow(01)$ 
Таблица истинности стохастических булевых функций $s_{1}(n+1)$ и $s_{2}(n+1)$ HКА $\boldsymbol{K}(\boldsymbol{S}, \boldsymbol{A})$ с четырьмя стохастическими дугами

\begin{tabular}{|c|c|c|c|c|c|c|}
\hline$a(n)$ & $s_{1}(n)$ & $s_{2}(n)$ & \multicolumn{2}{|c|}{$s_{1}(n+1)$} & \multicolumn{2}{c|}{$s_{2}(n+1)$} \\
\hline 0 & 0 & 0 & \multicolumn{2}{|c|}{0} & \multicolumn{2}{|c|}{0} \\
\hline 0 & 0 & 1 & \multicolumn{2}{|c|}{0} & \multicolumn{2}{|c|}{0} \\
\hline 0 & 1 & 0 & \multicolumn{2}{|c|}{0} & \multicolumn{2}{|c|}{0} \\
\hline 0 & 1 & 1 & 1 & 0 & 0 & 1 \\
\cline { 4 - 7 } & & $q_{1}$ & $q_{2}$ & $q_{1}$ & $q_{2}$ \\
\hline 1 & 0 & 0 & 1 & 0 & 0 & 1 \\
\hline 1 & 0 & 1 & \multicolumn{2}{|c|}{1} & \multicolumn{2}{|c|}{1} \\
\hline 1 & 1 & 0 & \multicolumn{2}{|c|}{1} & \multicolumn{2}{|c|}{1} \\
\hline 1 & 1 & 1 & \multicolumn{2}{|c|}{1} & \multicolumn{2}{|c|}{1} \\
\hline
\end{tabular}

Далее рассмотрим три случая сочетания стохастической природы дуг орграфа, представленные недетерминированными конечными автоматами.

При детерминированной диспетчеризации входных заявок с протоколом (1) имеем вероятности $p_{1}=1, p_{2}=0$. Тогда получим для $a=a(n), s_{1}=$ $=s_{1}(n), s_{2}=s_{2}(n)$ нелинейные нестационарные рекурсивные стохастические булевы функции в правой части уравнений состояний $\operatorname{HKA} K(S, A)$ с двумя оставшимися недетерминированными переходами $(11) \rightarrow(10)$ и $(11) \rightarrow(01)$ :

$$
\begin{aligned}
& s_{1}(n+1)=\begin{array}{|c|c|}
\hline a \oplus s_{1} s_{2} \oplus a s_{1} s_{2} & a \\
\hline q_{1} & q_{2} \\
\hline
\end{array}, \\
& s_{2}(n+1)=\begin{array}{|c|c|}
\hline a \oplus a s_{1} \oplus a s_{1} s_{2} & a \oplus s_{1} s_{2} \oplus a s_{1} \\
\hline q_{1} & q_{2} \\
\hline
\end{array} .
\end{aligned}
$$

Из (2) следует линейное явное (то есть не рекурсивное) детерминированное (то есть не стохастическое) нестационарное необходимое, но не достаточное условие

$$
a(n) s_{1}(n+1)=\begin{array}{|c|c|}
\hline a(n) & a(n) \\
\hline q_{1} & q_{2} \\
\hline
\end{array}
$$

эквивалентное числовому линейному явному нестационарному неравенству $s_{1}(n+1) \geqslant a(n)$, которое легко усмотреть из сравнения соответствующих столбцов табл. 2.

Аналогично, из (3) получим нелинейное рекурсивное нестационарное необходимое, но не достаточное стохастическое условие

$$
s_{1}(n) s_{2}(n+1)=\begin{array}{|c|c|}
\hline a(n) s_{1}(n) s_{2}(n) & s_{1}(n) s_{2}(n) \\
\hline q_{1} & q_{2} \\
\hline
\end{array}
$$

из которого следует нелинейное (квадратичное) рекурсивное детерминированное нестационарное необходимое, но не достаточное условие

$$
a(n) s_{1}(n) s_{2}(n+1)=\begin{array}{|c|c|}
\hline a(n) s_{1}(n) s_{2}(n) & a(n) s_{1}(n) s_{2}(n) \\
\hline q_{1} & q_{2} \\
\hline
\end{array}=a(n) s_{1}(n) s_{2}(n),
$$

эквивалентное утверждению $a(n) s_{1}(n)=1 \Rightarrow s_{2}(n+1)=s_{2}(n)$. Это линейное равенство также легко усмотреть из сравнения соответствующих столбцов и строк табл. 2. 
Таблица 3

Матрица переходов ДКА $K\left(S, A_{1}\right)$

\begin{tabular}{|c|c|c|c|}
\hline$S \backslash A_{1}$ & 00 & 01 & 10 \\
\hline$(00)$ & $(00)$ & $(00)$ & $(10)$ \\
\hline$(01)$ & $01)$ & $(00)$ & $(11)$ \\
\hline$(10)$ & $(00)$ & $(10)$ & $(11)$ \\
\hline$(11)$ & $(01)$ & $(10)$ & $(11)$ \\
\hline
\end{tabular}

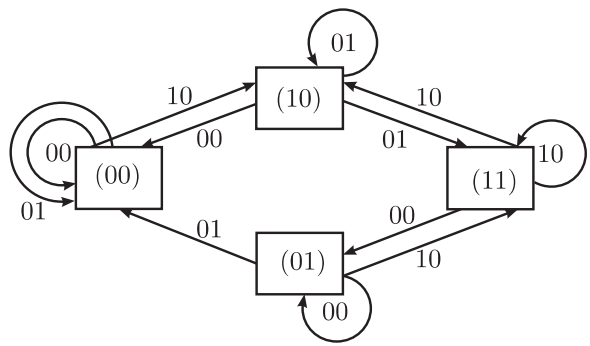

Рис. 2. Граф переходов ДКА $K\left(S, A_{1}\right)$

Избавимся от стохастичности дуг $(11) \rightarrow(10)$ и $(11) \rightarrow(01)$ с помощью изоморфного детерминированного конечного автомата (ДКА) $K\left(S, A_{1}\right)$ со входным алфавитом $A_{1}=\{00,01,10\}$, обозначив буквой 00 сигнал освобождения канала 1 (в том числе «холостой ход» при простое (01) этого канала), буквой 01 - сигнал освобождения канала 2 (в том числе «холостой ход» при простое (10) этого канала), буквой 10 - сигнал прихода входной заявки. Отношение вероятностей появления букв 00 и 01 во входном потоке сигналов ДКА $K\left(S, A_{1}\right)$ равно $q_{1} / q_{2}$. Соответствующим образом заменим матрицу и граф переходов ДКА $K\left(S, A_{1}\right)$ (см. табл. 3 и рис. 2$)$.

Отметим отсутствие повторов входных сигналов в разметке дуг, выходящих из одной вершины ДКА $K\left(S, A_{1}\right)$, в отличие от случая $\mathrm{HKA} K(S, A)$ (ср. рис. 1).

Тогда рекурсивную (то есть разрешимую последовательно) систему линейных рекурсивных нестохастических уравнений состояний автомата $K\left(S, A_{1}\right)$ получим из соответствующей таблицы истинности при входном сигнале $a_{1}(n) a_{2}(n)=a_{1} a_{2} \in A_{1}$ :

$$
\begin{gathered}
s_{1}(n+1)=a_{1} \oplus a_{1} a_{2} \oplus a_{2} s_{1} \oplus a_{1} a_{2} s_{1}=\alpha_{n} \oplus \beta_{n} s_{1}(n), \\
s_{2}(n+1)=a_{1} s_{1} \oplus a_{1} a_{2} s_{1} \oplus a_{2} s_{2} \oplus s_{2} \oplus a_{1} a_{2} s_{1} s_{2}=\gamma_{n} \oplus \delta_{n} s_{2}(n),
\end{gathered}
$$

где $\alpha_{n}:=a_{1} \overline{a_{2}}, \beta_{n}:=\overline{a_{1}} a_{2}, \gamma_{n}:=a_{1} \overline{a_{2}} s_{1}, \delta_{n}:=\overline{a_{2}} \oplus a_{1} a_{2} s_{1}$.

Рекурсивность системы (4), (5) проявляется в зависимости параметров уравнения (5) от решения уравнения (4). Из (4) и (5) получим явный вид уравнений состояний:

$$
\begin{aligned}
& s_{1}(n+1)=\sum_{t=0}^{n} \alpha_{n-t} \prod_{\tau=0}^{t-1} \beta_{n-\tau} \oplus s_{1}(0) \prod_{\tau=0}^{n} \beta_{n-\tau}, \\
& s_{2}(n+1)=\sum_{t=0}^{n} \gamma_{n-t} \prod_{\tau=0}^{t-1} \delta_{n-\tau} \oplus s_{2}(0) \prod_{\tau=0}^{n} \delta_{n-\tau} .
\end{aligned}
$$

Здесь для универсальности обозначений предполагается равенство

$$
\prod_{\tau=0}^{-1} \beta_{n-\tau}:=1
$$

Поскольку $\prod_{\tau=0}^{n} \beta_{n-\tau}=0$, если $\exists k \in \overline{0, n}: \beta_{k}=\overline{a_{1}(k)} a_{2}(k)=0 ; \prod_{\tau=0}^{n} \delta_{n-\tau}=$ $=0$, если $\exists k \in \overline{0, n}: \delta_{k}=\overline{a_{2}(k)} \oplus a_{1}(k) a_{2}(k) s_{1}(k)=0$, то явные уравнения 
состояний (6) и (7) примут окончательный вид:

$s_{1}(n+1)=\left\{\begin{array}{l}\sum_{t=0}^{n} \alpha_{n-t} \oplus s_{1}(0) \leftarrow\left(\forall k \in \overline{0, n} \Rightarrow \beta_{k}=\overline{a_{1}} a_{2}=1\right), \\ \sum_{t=0}^{m-1} \alpha_{n-t} \leftarrow(m \leqslant n) \wedge\left(\forall k \in \overline{0, m-1} \Rightarrow \beta_{k}=\overline{a_{1}} a_{2}=1\right) \wedge\left(\beta_{m}=0\right) ;\end{array}\right.$
$s_{2}(n+1)=\left\{\begin{array}{l}\sum_{t=0}^{n} \gamma_{n-t} \oplus s_{2}(0) \leftarrow\left(\forall k \in \overline{0, n} \Rightarrow \delta_{k}=\overline{a_{2}} \oplus a_{1} a_{2} s_{1}=1\right), \\ \sum_{t=0}^{m-1} \gamma_{n-t} \leftarrow(m \leqslant n) \wedge\left(\forall k \in \overline{0, m-1} \Rightarrow \delta_{k}=1\right) \wedge \\ \wedge\left(\delta_{m}=\overline{a_{2}} \oplus a_{1} a_{2} s_{1}=0\right) .\end{array}\right.$

Из (4) следует необходимое, но не достаточное условие

$$
a_{1}(n) s_{1}(n+1)=a_{1}(n) \oplus a_{1}(n) a_{2}(n)=a_{1}(n) \overline{a_{2}(n)}
$$

Аналогично, из (5) получим необходимое, но не достаточное условие

$$
a_{2}(n) s_{2}(n+1)=a_{2}(n) a_{1}(n) s_{1}(n) s_{2}(n) .
$$

СMO со стохастической диспетчеризацией входных заявок и выработкой сигналов на освобождение приборов. В общем случае стохастического поведения пропускной способности дуг, выходящих из вершин (00) и (11), уравнения перехода зависят от совместного распределения вероятностей переходов по этим четырём дугам. Из табл. 2 получим рекурсивные стохастические булевы функции в правых частях уравнений состояний HKA

$$
\begin{aligned}
& s_{1}(n+1)=\begin{array}{|c|c|c|c|}
\hline a \oplus s_{1} s_{2} \oplus a s_{1} s_{2} & a & s_{1} s_{2} \oplus a s_{1} \oplus a s_{2} & a s_{1} \oplus a s_{2} \oplus a s_{1} s_{2} \\
\hline q_{1} p_{1} & q_{2} p_{1} & q_{1} p_{2} & q_{2} p_{2} \\
\hline
\end{array} \\
& s_{2}(n+1)=\begin{array}{|c|c|c|c|}
\hline a \oplus a s_{1} \oplus a s_{1} s_{2} & a \oplus s_{1} s_{2} \oplus a s_{1} & a & a \oplus s_{1} s_{2} \oplus a s_{1} s_{2} \\
\hline q_{1} p_{1} & q_{2} p_{1} & q_{1} p_{2} & q_{2} p_{2} \\
\hline
\end{array} .
\end{aligned}
$$

Следует заметить, что произведение $q_{i} p_{j}$ не означает вероятность одновременного появления двух сигналов 0 и 1, а лишь исчисляет вероятность, что в данный момент времени при поступлении сигнала 1 заявка отправилась бы на $j$-тый прибор, а при поступлении сигнала 0 сработал бы $i$-тый прибор.

Построим изоморфный ДКА $K\left(S, A_{2}\right)$ со входным алфавитом $A_{1}=\{00,01,10,11\}$, обозначив буквой 00 сигнал освобождения канала 1 (в том числе «холостой ход» при простое (01) этого канала), буквой 01 - сигнал освобождения канала 2 (в том числе «холостой ход» при простое (10) этого канала), буквой 10 - сигнал прихода входной заявки, которую может обработать только канал 1 , буквой 11 - сигнал прихода входной заявки, которую может обработать только канал 2. Отношение вероятностей появления букв 00 и 01 во входном потоке сигналов ДКА $K\left(S, A_{2}\right)$ равно $q_{1} / q_{2}$, а отношение вероятностей появления букв 10 и 11 во входном потоке сигналов равно $p_{1} / p_{2}$. Соответствующим образом заменим граф переходов (см. рис. 3).

Петли на орграфе вновь являются детерминированными, и переход по ним осуществляется при поступлении подходящего входного сигнала (ср. рис. 1). 


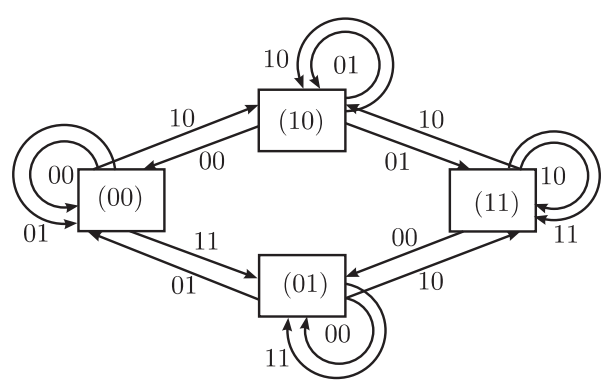

Рис. 3. Граф переходов ДКА $K\left(S, A_{2}\right)$

Тогда уравнения состояний автомата $K\left(S, A_{2}\right)$ получим из соответствующей таблицы истинности при входной букве $a_{1}(n) a_{2}(n)=a_{1} a_{2} \in A_{2}$.

Аналогично первому случаю составим таблицу истинности детерминированных булевых функций $s_{1}(n+1)$ и $s_{2}(n+1)$ ДКА $K\left(S, A_{2}\right)$ и выведем из их СДНФ линейные независимые рекурсивные детерминированные уравнения состояний

$$
\begin{aligned}
& s_{1}(n+1)=a_{1} \oplus a_{1} a_{2} \oplus a_{2} s_{1}=\alpha(n) \oplus \beta(n) s_{1}(n), \\
& s_{2}(n+1)=s_{2} \oplus a_{2} s_{2} \oplus a_{1} a_{2}=\gamma(n) \oplus \delta(n) s_{2}(n),
\end{aligned}
$$

где $\alpha(n) \stackrel{\text { def }}{=} a_{1} \overline{a_{2}}, \beta(n) \stackrel{\text { def }}{=} a_{2}, \gamma(n) \stackrel{\text { def }}{a_{1}} a_{2}, \delta(n) \stackrel{\text { def }}{=} \overline{a_{2}}$.

Из (8) и (9) окончательно получим явный вид уравнений состояний (6) и (7):

$$
\begin{aligned}
& s_{1}(n+1)=\left\{\begin{array}{c}
\sum_{t=0}^{n} \alpha(n-t) \oplus s_{1}(0) \leftarrow\left(\forall k \in \overline{0, n} \Rightarrow \beta(k)=a_{2}(k)=1\right), \\
\sum_{t=0}^{m-1} \alpha(n-t) \leftarrow(m \leqslant n) \wedge\left(\forall k \in \overline{0, m-1} \Rightarrow \beta(k)=a_{2}(k)=1\right) \wedge \\
\wedge\left(\beta(m)=a_{2}(m)=0\right) ;
\end{array}\right. \\
& s_{2}(n+1)=\left\{\begin{aligned}
\sum_{t=0}^{n} \gamma(n-t) \oplus s_{2}(0) \leftarrow\left(\forall k \in \overline{0, n} \Rightarrow \delta(k)=\overline{a_{2}(k)}=1\right), \\
\sum_{t=0}^{m-1} \gamma(n-t) \leftarrow(m \leqslant n) \wedge\left(\forall k \in \overline{0, m-1} \Rightarrow \delta(k)=\overline{a_{2}(k)}=1\right) \wedge \\
\wedge\left(\delta(m)=\overline{a_{2}}(m)=0\right) .
\end{aligned}\right.
\end{aligned}
$$

Из (8) следует необходимое, но не достаточное линейное условие

$$
a_{2}(n) s_{1}(n+1)=a_{2}(n) s_{1}(n),
$$

эквивалентное утверждению $a_{2}(n)=1 \Rightarrow s_{1}(n+1)=s_{1}(n)$, которое легко усмотреть из сравнения соответствующих столбцов и строк таблицы истинности.

Аналогично, из (9) получим необходимое, но не достаточное линейное условие

$$
a_{2}(n) s_{2}(n+1)=a_{2}(n) a_{1}(n),
$$

эквивалентное утверждению $a_{2}(n)=1 \Rightarrow s_{2}(n+1)=a_{1}(n)$, которое также легко усмотреть из сравнения соответствующих столбцов и строк таблицы истинности. 
СМО со стохастической диспетчеризацией и детерминированной выработкой сигналов на освобождение приборов. При недетерминированной диспетчеризации входных заявок с вероятностью $p_{1}<1$ перехода по оптимальной дуге $(00) \rightarrow(01)$ и вероятностью $p_{2}=1-p_{1}>0$ перехода по неоптимальной дуге $(00) \rightarrow(01)$ введём детерминированную диспетчеризацию выходных заявок: $q_{1}=1$, $q_{2}=0$. Матрица переходов $\mathrm{HKA} K(S, A)$ дана в табл. 4.

Из таблицы истинности стохастических булевых функций $s_{1}(n+1)$ и $s_{2}(n+1) \mathrm{HKA}$ $K(S, A)$ с двумя стохастическими дугами $(00) \rightarrow(10)$ и $(00) \rightarrow(01)$ получим нелинейные нестационарные рекурсивные стохастические булевы функции в правой части уравнений состояний $\mathrm{HKA} K(S, A)$ с двумя оставшимися недетерминированными пере-

Таблица 4 ходами $(00) \rightarrow(10)$ и $(00) \rightarrow(01):$

$$
\begin{aligned}
& s_{1}(n+1)=\begin{array}{|c|c|}
\hline a \oplus s_{1} s_{2} \oplus a s_{1} s_{2} & a s_{1} \oplus a s_{2} \oplus s_{1} s_{2} \\
\hline p_{1} & p_{2} \\
\hline
\end{array} \\
& s_{2}(n+1)=\begin{array}{|c|c|}
\hline a s_{1} \oplus a s_{2} \oplus a s_{1} s_{2} & a \\
\hline p_{1} & p_{2} \\
\hline
\end{array} .
\end{aligned}
$$

Из (10) следуют линейные детерминированные нестационарные необходимые, но не достаточные условия

$$
\begin{aligned}
& a(n) s_{1}(n) s_{1}(n+1)=\begin{array}{|c|c|}
\hline a(n) s_{1}(n) & a(n) s_{1}(n) \\
\hline p_{1} & p_{2} \\
\hline a(n) s_{2}(n) & a(n) s_{2}(n) \\
\hline p_{1} & p_{2} \\
a(n) s_{2}(n) s_{1}(n+1)=a(n) s_{1}(n),
\end{array}=a(n) s_{2}(n),
\end{aligned}
$$

эквивалентные утверждению $\left(a(n) s_{1}(n)=1\right) \vee\left(a(n) s_{2}(n)=1\right) \Rightarrow s_{1}(n+1)=1$, которое можно также усмотреть из сравнения соответствующих столбцов и строк таблицы истинности.

Аналогично, из (11) получим линейное детерминированное нестационарное необходимое, но не достаточное условие

$$
s_{1}(n) s_{2}(n+1)=\begin{array}{|c|c|}
\hline a(n) s_{1}(n) & a(n) s_{1}(n) \\
\hline p_{1} & p_{2} \\
\hline
\end{array}=s_{1}(n) a(n),
$$

эквивалентное утверждению $s_{1}(n)=1 \Rightarrow s_{2}(n+1)=a(n)$. Это равенство также легко усмотреть из сравнения соответствующих столбцов и строк таблицы истинности.

Избавимся от стохастичности дуг $(00) \rightarrow(10)$ и $(00) \rightarrow(01)$ с помощью изоморфного ДКА $K\left(S, A_{3}\right)$ со входным алфавитом $A_{3}=\{00,10,11\}$, обозначив буквой 00 сигнал выхода обработанной заявки (в том числе «холостой 
Таблица 5 ход» при простое (00) системы), буквой Матрица переходов ДКА $\boldsymbol{K}\left(\boldsymbol{S}, \boldsymbol{A}_{\mathbf{3}}\right) \quad 10$ - сигнал прихода входной заявки, ко-

\begin{tabular}{|c|c|c|c|}
\hline & 00 & 10 & 11 \\
\hline$(00)$ & $(00)$ & $(01)$ & $(10)$ \\
\hline$(01)$ & $(00)$ & $(01)$ & $(11)$ \\
\hline$(10)$ & $(00)$ & $(11)$ & $(10)$ \\
\hline$(11)$ & $(10)$ & $(11)$ & $(11)$ \\
\hline
\end{tabular}

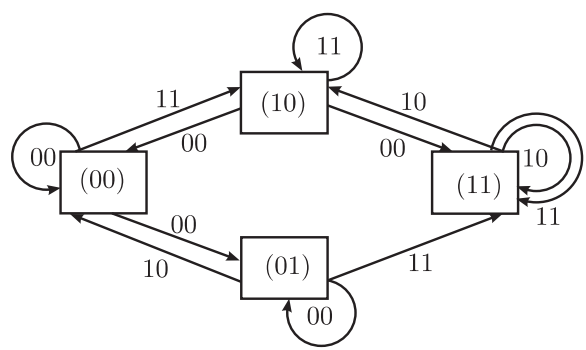

Рис. 4. Граф переходов ДКА $K\left(S, A_{3}\right)$ торую может обслужить только канал 2 (в том числе отказ (01) при занятости этого канала или общий отказ (11) всей СМО), буквой 11 - сигнал прихода входной заявки, которую может обслужить только канал 1 (в том числе отказ (10) при занятости этого канала или общий отказ (11) всей СМО). Отношение вероятностей появления букв 11 и 10 во входном потоке сигналов ДКА $K\left(S, A_{3}\right)$ равно $p_{1} / p_{2}$ при условии $p_{1}+$ $+p_{2}=1$. Соответствующим образом заменим матрицу и граф переходов ДКА $K\left(S, A_{3}\right)$ (см. табл. 5 и рис. 4$)$.

Отметим отсутствие повторов входных сигналов в разметке дуг, выходящих из одной вершины ДКА $K\left(S, A_{3}\right)$, в отличие от случая $\mathrm{HKA} K(S, A)$ (ср. рис. 4$)$.

Тогда уравнения состояний автомата $K\left(S, A_{3}\right)$ получим из таблицы истинности детерминированных булевых функций $s_{1}(n+1)$ и $s_{2}(n+1)$ при входном сигнале $a_{1}(n) a_{2}(n)=a_{1} a_{2} \in A_{3}$ :

$$
\begin{gathered}
s_{1}(n+1)=a_{1} a_{2} \oplus a_{1} s_{1} \oplus a_{1} a_{2} s_{1} \oplus s_{1} s_{2} \oplus a_{1} s_{1} s_{2} \oplus a_{2} s_{1} s_{2} \oplus a_{1} a_{2} s_{1} s_{2}= \\
=\alpha(n) \oplus \beta(n) s_{1}(n), \\
s_{2}(n+1)=a_{1} \oplus a_{1} a_{2} \oplus a_{1} a_{2} s_{2}=\gamma(n) \oplus \delta(n) s_{2}(n),
\end{gathered}
$$

где $\alpha(n) \stackrel{\text { def }}{=} a_{1}(n) a_{2}(n), \beta(n) \stackrel{\text { def }}{=} \overline{a_{2}}(n)\left[a_{1}(n) \oplus \overline{a_{1}(n)} s_{2}(n)\right], \gamma(n) \stackrel{\text { def }}{=} a_{1}(n) \overline{a_{2}(n)}$, $\delta(n) \stackrel{\text { def }}{=} a_{1}(n) a_{2}(n)$.

Из (12) и (13) окончательно получим явный вид уравнений состояний

$$
\begin{aligned}
& s_{1}(n+1)=\left\{\begin{array}{l}
\sum_{t=0}^{n} \alpha(n-t) \oplus s_{1}(0) \leftarrow \\
\left(\forall k \in \overline{0, n} \Rightarrow \beta(k)=\overline{a_{2}(k)}\left[a_{1}(k) \oplus \overline{a_{1}(k)} s_{2}(k)\right]=1\right), \\
\sum_{t=0}^{m-1} \alpha(n-t) \leftarrow(m \leqslant n) \wedge(\forall k \in \overline{0, m-1} \Rightarrow \beta(k)=1) \wedge(\beta(m)=0) ;
\end{array}\right. \\
& s_{2}(n+1)=\left\{\begin{array}{l}
\sum_{t=0}^{n} \gamma(n-t) \oplus s_{2}(0) \leftarrow\left(\forall k \in \overline{0, n} \Rightarrow \delta(k)=a_{1}(k) a_{2}(k)=1\right), \\
\sum_{t=0}^{m-1} \gamma(n-t) \leftarrow(m \leqslant n) \wedge(\forall k \in \overline{0, m-1} \Rightarrow \delta(k)=1) \wedge(\delta(m)=0) .
\end{array}\right.
\end{aligned}
$$

Из (12) следует необходимое, но не достаточное линейное условие

$$
a_{1}(n) a_{2}(n) s_{1}(n+1)=a_{1}(n) a_{2}(n),
$$


эквивалентное утверждению $a_{1}(n) a_{2}(n)=1 \Rightarrow s_{1}(n+1)=1$, которое легко усмотреть из сравнения соответствующих строк и столбцов таблицы истинности.

Аналогично, из (13) также получим необходимое, но не достаточное линейное условие

$$
s_{2}(n) s_{2}(n+1)=s_{2}(n) a_{1}(n),
$$

эквивалентное утверждению $s_{2}(n)=1 \Rightarrow s_{2}(n+1)=a_{1}(n)$, которое легко усмотреть из соответствующей таблицы истинности.

Заключение. Выкладки аналогично переносятся на случай большего числа каналов обслуживания, наличия очередей, а также совместного стохастического поведения параметров каналов обслуживания. Несмотря на значительное усложнение неклассического орграфа СМО, явный вид уравнений переходов состояний позволяет проводить имитационное моделирование в случае как простейших, так и произвольных случайных процессов поступления и обработки заявок.

Рост ёмкости очереди приводит к увеличению $r$-значности поставленной задачи, которая легко может быть снижена до $r=2$ (подобно рассматриваемому случаю) за счёт увеличения длины вектора $S_{i}$, описывающего $i$-тое состояние СМО в разработанной авторами нотификации.

Отметим, что такие среды автоматизированного динамического моделирования систем, как GPSS (включая GPSS World) и Simulink, не располагают удовлетворительным инструментарием моделирования описанных в работе $\mathrm{CMO}$, в частности, они не позволяют учитывать предложенную авторами диспетчеризацию поступающих заявок. Разработанные авторами нотификация состояний и алгоритм получения уравнений состояния подобных систем позволяют:

1) при необходимости распространить его на другие типы протоколов диспетчеризации;

2) автоматизировать процесс получения уравнений состояний;

3) моделировать описанные СМО практически в любой программной среде, допускающей работу с булевой алгеброй.

На основе разработанной нотификации, в частности, авторами был создан комплекс программ, полностью автоматизирующий построение, разметку и визуализацию графа состояний подобных СМО для произвольного числа каналов и мест в очередях к ним.

\section{БИБЛИОГРАФИЧЕСКИЙ СПИСОК}

1. Артамонов Г. Т, Брехов О. М. Аналитические вероятностные модели функционирования ЭВМ. М.: Энергия, 1978. 368 с. [Artamanov G. T., Brehov O. M. Analytic probability models of the functioning of computers. Moscow: Énergiya, 1978. 368 pp.]

2. Введенская Н. Д., Добрушин Р. Л., Карпелевич Ф. И. Система обслуживания с выбором наименьшей из двух очередей - асимптотический подход// Пробл. передачи информ., 1996. Т. 32, № 1. C. 20-34; англ. пер.: Vvedenskaya N. D., Dobrushin R. L., Karpelevich F. I. A queueing system with a choice of the shorter of two queues - an asymptotic approach // Problems Inform. Transmission, 1996. Vol. 32, no. 1. Pp. 15-27.

3. Введенская Н. Д. Большая система обслуживания с передачей сообщения по нескольким путям // Пробл. передачи информ., 1998. Т. 34, №2. С. 98-108; Vvedenskaya N. D. Large Queueing System where Messages are Transmitted via Several Routes // Problems Inform. Transmission, 1998. Vol. 34, no. 2. Pp. 180-189. 
4. Печинкин A.B., Таташев А.Г. Обобщение дисциплины преимущественного разделения процессора // Изв. АН СССР. Техн. кибернетика, 1981. № 4. С. 120-125; англ. пер.: Pechinkin A.V., Tatashev A.G. Generalization of the discipline of advantageous sharing of a processor // Eng. Cybern., 1981. Vol. 19, no. 4. Pp. 91-96.

5. Д'Апиче Ч., Манзо Р., Печинкин А.В. Система обслуживания $M A P_{K} / G_{K} / 1$ конечной емкости с обобщенной дисциплиной преимущественного разделения прибора // Автомат. и телемех., 2004. №11. C. 114-121; англ. пер.: D’Apice C., Manzo R., Pechinkin A.V. A finite $M A P_{K} / G_{K} / 1$ queueing system with generalized foregroundbackground processor-sharing discipline// Autom. Remote Control, 2004. Vol.65, no. 11. Pp. $1793-1799$.

6. Rykov V. V. Monotone Control of Queueing Systems with Heterogeneous Servers// Queueing Syst., 2001. Vol. 37, no.4. Pp. 391-403.

7. de Véricourt F., Zhou Y.-P. On the incomplete results for the heterogeneous server problem // Queueing Syst., 2006. Vol.52, no. 3. Pp. 189-191.

8. Nawijn W. M. On a two-server finite queuing system with ordered entry and deterministic arrivals // Eur. J. Oper. Res., 1984. Vol.18, no. 3. Pp. 388-395.

9. Elsayed E.A. Multichannel queueing systems with ordered entry and finite source// Comput. Oper. Res., 1983. Vol. 10, no. 3. Pp. 213-222.

10. Котенко А.П., Букаренко М.Б. Аналитическое описание систем массового обслуживания с использованием колец вычетов / В сб.: Труды седъмой Всероссийской научной конференции с международным участием. Часть 2 / Математическое моделирование и краевые задачи. Самара: СамГТУ, 2010. С. 136-140. [Kotenko A.P., Bukarenko M. B. Analytical description of queuing systems with use residue rings/ In: Proceedings of the Seventh All-Russian Scientific Conference with international participation. Part 2 / Matem. Mod. Kraev. Zadachi. Samara: Samara State Technical Univ., 2010. Pp. 136-140].

11. Букаренко М.Б. Система массового обслуживания с раздельными очередями к каналам/ В сб.: Современные проблемы математики: Тезисы 42-ой Всероссийской конференции. Екатеринбург: ИММ УрО РАН, 2011. С. 11-13. [Bukarenko M.B. A queuing system with separate queues to channels / In: Modern problems of mathematics. Ekaterinburg: IMM UrO RAN, 2011. Pp. 11-13].

Поступила в редакцию 06/VII/2012;

в окончательном варианте - 22/VIII/2012.

MSC: 60K25; 90B22, 68M20

\title{
A QUEUING SYSTEM WITH DISTINCT DEVICES AS THE FINITE STATE MACHINE
}

\section{A.P. Kotenko, M. B. Bukarenko}

Samara State Technical University,

244, Molodogvardeyskaya st., Samara, 443100, Russia.

E-mails: ako1959@mail.ru, maxim.bukarenko@gmail.com

\begin{abstract}
Queuing systems with distinct channels are considered. Channels may have different capacities (from each other) and distinct queues. The term "dispatch control" is introduced to optimize the system, considering the average time of service and failure probability minimization. These systems are treated as deterministic or nondeterministic finite state machines. State equations of these systems in the form of Zhegalkin polynomial are derived.
\end{abstract}

Key words: queuing system, finite state machine, distinct channels, dispatch control.

Original article submitted 06/VII/2012; revision submitted 22/VIII/2012.

Andrey P. Kotenko (Ph. D. (Phys. \& Math.)), Associate Professor, Dept. of Applied Mathematics \& Computer Science. Maksim B. Bukarenko, Postgraduate Student, Dept. of of Applied Mathematics \& Computer Science. 